\title{
Strong Angular Clustering of Very Blue Galaxies: Evidence of a Low Redshift Population
}

\author{
Stephen D. Landy ${ }^{1}$, Alexander S. Szalay ${ }^{2,3}$ and David C. Koo ${ }^{4}$
}

1. Carnegie Observatories, 813 Santa Barbara St.,Pasadena, CA 91101

2. Dept. of Physics and Astronomy, The Johns Hopkins University, 34th \& Charles St., Baltimore, MD 21218

3. Dept. of Physics, Eötvös University, Budapest, H-1088

4. UCO/Lick Observatory and Board of Studies in Astronomy and Astrophysics, University of California, Santa Cruz, CA 95064

\begin{abstract}
We have studied galaxy two-point angular correlations as a function of color using 4-m plate photometry in two independent fields. Each field consists of over 2900 galaxies with magnitudes $20<B_{J}<23.5$ in an area of approximately $750 \operatorname{arcmin}^{2}$. We find that the autocorrelation amplitude of the bluest $15 \%$ of galaxies is surprisingly strong, with a relative increase in clustering amplitude of a factor of 6 over that of the complete data set, while exhibiting a power law slope consistent with the canonical value of -0.8 . These very blue galaxies are also found to be weakly correlated with galaxies of median color and marginally anti-correlated with the reddest subset. These correlation properties are incompatible with existing simple models of the galaxy distribution; they suggest that a significant fraction, more than $50 \%$, of these very blue galaxies are a faint population which lie at nearby redshifts $z<0.3$.
\end{abstract}

Subject headings:

Cosmology: Large Scale Structure

Galaxies: Clustering, Distances and Redshifts, Evolution, Luminosity Function 


\section{INTRODUCTION}

The nature of faint field galaxies and their high surface density at faint magnitudes remain a mystery despite extensive new observations over the last 15 years (see Koo and Kron 1992, Lilly 1993, and Koo 1996 for recent reviews). Besides fainter number counts and colors, and more recently the morphology of faint galaxies from Hubble Space Telescope observations (Griffiths et al. 1994), the key new observations have been redshifts to limits as faint as $B_{J}=24$ for 12 galaxies (Cowie, Songaila, \& Hu 1991), and 73 galaxies (Glazebrook et al. 1995) and to $I=22$ for almost 600 galaxies (Lilly et al. 1995). The detailed conclusions of these deep redshift surveys remain at odds, though all agree that substantial evolution has occurred in the luminosity function of field galaxies (Lilly et al. 1995, Colless 1995). Whether such changes are caused by a new population, substantial mergers, or enhanced star-formation among dwarfs has yet to be resolved. Moreover, both of the larger surveys remain incomplete at the $20 \%$ to $27 \%$ level, with the bulk of the unidentified galaxies being bluer than average. This level of incompleteness is high enough to make a significant difference in understanding the nature of faint galaxies, especially if the bulk of the unidentified population is either at high or low redshifts.

This paper places additional constraints on the nature of the bluest galaxies by using another observable property of faint galaxies, namely, their angular clustering behavior. Although the approach of using angular correlation functions to study galaxy evolution is not new (Koo \& Szalay 1984) and has been applied to several samples of faint galaxies, at different wavelengths, or for gross cuts in color (Efstathiou et al. 1991, Bernstein et al. 1994, Infante \& Pritchet 1995, Neuschaefer \& Windhorst 1995), none of the analyses so far have explored the variation of clustering among galaxies of extreme colors. Here we use the angular correlation function as a tool to measure, or at least constrain, the redshift distribution of such galaxies.

The two-point angular correlation function $w(\theta)$ is well known and defined by the joint probability $\delta P$ of finding galaxies in both of the elements of solid angle $\delta \Omega_{1}$ and $\delta \Omega_{2}$ placed at an angular separation $\theta$,

$$
\delta P=N^{2}[1+w(\theta)] \delta \Omega_{1} \delta \Omega_{2}
$$

where $N$ is the mean surface density of galaxies. The observations are usually close to a power law,

$$
w(\theta)=A_{w} \theta^{1-\gamma},
$$

with amplitude $A_{w}$ and $\gamma \sim 1.8$ (see Peebles 1980). As an estimator for this function we will use that described in Landy \& Szalay (1993) for its improved error properties.

The scaling of angular correlations as a function of limiting magnitude is well understood: as the limiting magnitude of a survey increases, there are more projected pairs that weakens the correlations (Peebles 1980), and the amplitude is proportional to an integral over the second moment of $d n / d z$, the redshift distribution of the galaxies,

$$
A_{w} \propto \int d z c(z)\left(\frac{d n}{d z}\right)^{2} /\left[\int d z\left(\frac{d n}{d z}\right)\right]^{2}
$$


where $c(z)$ depends on evolution, curvature, and $\gamma$ (see e.g. Koo \& Szalay 1984). Less is known about changes due to color selection. Differences in the measured clustering as a function of angle $w(\theta)$, for subsamples such as those chosen by color, can occur for two reasons: each subsample may be dominated by a different population with different clustering properties, and/or different redshift distributions. This degeneracy between intrinsic clustering and redshift distributions can be broken by considering other information such as the overall shape of the correlation function, cross-correlations with known populations, and number counts. Correlation functions are normally not thought of as a probe of redshift distributions, but as pointed out earlier (Koo and Szalay 1984), angular correlation functions provide powerful constraints on this distribution (and hence evolution and cosmology), which is particularly valuable in the absence of spectroscopic redshifts for very faint samples, for example, to $I>26$. Thus the method serves as an independent check or constraint on models that attempt to describe the more easily measured colors and counts of faint galaxies.

Since early-type (E/SO/Sa) galaxies have stronger than average intrinsic clustering (Giovanelli, Haynes, \& Chincarini 1986), the a priori expectation is for red galaxies to have correlation amplitudes higher than that of an average field sample. In contrast, late-type, blue galaxies today appear to be more weakly clustered than an average field sample (Nicotra, Abadi, \& Lambas 1993). Some groups have proposed that such galaxies were brighter in the past because of enhanced star formation (Broadhurst, Ellis, \& Shanks 1988). If so, their redshift distributions will be more extended than without this luminosity increase. In this case, one might expect a further reduction in clustering amplitude for blue galaxies observed to fainter limiting magnitudes. It has also been recently reported that low surface brightness galaxies are more weakly clustered than the general population (Neuschaefer \& Windhorst 1995), also expected in a CDM dominated universe (Efstathiou 1995).

To compare these expectations with observations, we have calculated the correlation functions of subsets of galaxies chosen as a function of color: $U-B_{J}, U-R_{F}$, and $B_{J}-R_{F}$. We start with extreme subsets of either red or blue objects and then increase the range to include objects of less extreme color. Also calculated is the correlation function for samples selected in bands of color as well as the cross correlation between samples selected in different bands. We report results on $U-R_{F}$, as this color gives the largest wavelength range to discriminate color-dependent effects. In addition, this is the combination for which the galaxy colors are least explicitly dependent on their redshift, and thus most correlated with intrinsic color (Koo 1986). The correlation amplitude dependence on $U-B_{J}$ and $B_{J}-R_{F}$ is similar to $U-R_{F}$, although the enhancements were weaker.

\section{DATA}

The photometry data are from two fields of approximately $750 \operatorname{arcmin}^{2}$ each and based on photographic plates taken at the prime focus of the Mayall $4 \mathrm{~m}$ telescope at Kitt Peak National Observatory. One field SA57 $(1306+2939)$ is at the North Galactic Pole; the other field SA68 $(0015+1537)$ has galactic coordinates of $l=-111$ and $b=-46$ and

is near Perseus-Pisces. Details of the observations and data reduction techniques have been published elsewhere (Kron 1980, Koo 1986). Here it is sufficient to note that the 
plates were digitized using the Berkeley PDS microdensitometer; that areas in the fields around bright stars as well as bad pixel lines in the data reduction have been excised from the fields; that both fields are considered complete to $B_{J}=23.5$, and finally that two plates were used in each bandpass with only multiple detections included in the catalogs to decrease the incidence of artifacts. We have selected galaxies in the magnitude range $20<B_{J}<23.5$, with 2922 galaxies in SA57 and 2999 galaxies in SA68.

The correlation properties of the same data have previously been analyzed, but only as a function of $B_{J}$ magnitude (Koo \& Szalay 1984). This earlier work is in agreement with that of other researchers (Stevenson et al. 1985; Pritchet \& Infante 1992; Jones, Shanks, \& Fong 1988; Bernstein et al. 1994; Brainerd, Smail, \& Mould 1995 ), who found that the scaled two-point angular correlation function amplitude is consistent with that found locally, except for hints of mild evolution in luminosity and/or clustering.

Given the unexpected nature of our results (see below) we have performed numerous checks to determine the validity of our measurements. It should, however, be emphasized that these data are derived from multiple plates and that the results are qualitatively identical for the three independent color combinations for both red and blue galaxies in two separate fields. The similarity of results decreases the probability that artifacts, such as gradients across the plates in different passbands, are responsible for the signal. As a further check against artifacts caused by gross variations in color over the plates, the correlation analyses were rerun with the median $U-R_{F}$ color equilibrated over the plates by use of a 400" x400" square smoothing window. Although this adjustment, which amounted to several tenths of magnitude in some areas, weakened the signals, all general trends were still clearly evident. Additional tests were run on the data to check whether either population was associated with bright objects such as galaxies or stars in the fields. These proved negative. Cross correlations between stars and galaxies in the fields also indicated no obvious problems with the catalogs as did analysis using a subset of stars identified through proper motion analysis.

\section{Results}

\subsection{Measured Angular Correlation Amplitudes}

The correlation amplitudes for SA57 and SA68, as a function of $U-R_{F}$ color, are shown in Figure 1, together with plots showing the percentage of data included in each subset. These results show the angular correlation amplitude of the reddest and bluest subsets of galaxies, respectively. The horizontal line is the correlation amplitude of the whole sample. In order to present a single number, an amplitude, the correlation function was integrated between 9 and 144 arcsecs to reduce noise and for compatibility with the earlier work (Koo \& Szalay 1984). Assuming a nominal redshift of $0.3, H_{o}=50 \mathrm{~km}$ $\mathrm{sec}^{-1} \mathrm{Mpc}^{-1}$, and $q_{o} \sim 0$ these limits span $70 \mathrm{kpc}$ to $1.1 \mathrm{Mpc}$. The amplitude of $w(\theta)$, $A_{w}$, is reported using the value of -0.8 for the power law slope at one degree in units of $10^{-4}$. For the average of the two fields we obtained $A_{w}($ all $)=18.0 \pm 1.8$. Statistical (Poisson) error bars are shown for all results, although these certainly underestimate the true variance in the signal, as will be discussed below.

Both fields show a marked enhancement in correlation amplitude, with increases 
greater than a factor of ten for the extreme subsets in both red and blue. As a check against the possibility that only the most extreme galaxies contribute to the rise in amplitude (because of artifacts in the data), we calculated $w(\theta)$ in bands of color for both the red and blue galaxies. The results are shown in Figure 2. It is evident that the increase in angular correlation function exists over more than one magnitude in color, which indicates that the enhancement is genuine.

Figure 3 shows $w(\theta)$ for 'red' galaxies $U-R_{F}>1.5$ and 'blue' galaxies $U-R_{F}<-0.25$ together with the entire data set for both fields in five bins of equal logarithmic width between 18" and 289". These results are compared against a fiducial no-evolution model assuming a power law index of $-0.8, q_{o}=0.01$, and $H_{o}=50 \mathrm{~km} / \mathrm{sec}$. Both the red and blue subsets in each field show qualitative agreement with a power law slope of -0.8 , together with enhanced clustering over this entire range.

Other recent correlation function studies (Efstathiou et al. 1991; Brainerd et al. 1995; Neuschaefer et al. 1995) that used CCD detectors have found quite weak angular correlation functions for objects with an isophotal magnitude $24<B_{J}<26$. When the Efstathiou et al. (1991) sample was divided into red and blue subsets, the blue subset was found to have a marginally $(<2 \sigma)$ stronger correlation amplitude. One of their fields, SA68, overlaps with one of ours. When we apply a similar color split to our data as adopted by these authors, we obtain similarly low amplitudes (as seen on Figure 1). Only with more extreme color cuts do the enhancements in correlation amplitude become evident.

Our error bars correspond to the statistical noise in the LS estimator (Landy \& Szalay 1993), i.e. we assume that the distribution of the total number of galaxies in the field is Poisson, thus the variance in the correlation is Poisson in the bin counts. The LS estimator, conditional on the number of galaxies observed, is optimal in this case, and still minimizes the variance in all the other cases. If, however, there is additional large scale clustering (as shown by the presence of a cluster), the irreducible higher order moments of the galaxy counts on scales of the whole field will result in additional variance. An a priori determination of this variance, on the other hand, requires knowledge of these higher order moments at these magnitudes, colors, and angular scales. As these are not available, we only plot the Poisson errors, but expect the full error to be about a factor of two higher.

Table 1

\begin{tabular}{cccc}
\hline \multicolumn{4}{c}{ Auto and Cross-Correlations by Bands of Color } \\
\hline & Blue (20\%) & Mid (60\%) & Red (20\%) \\
\hline Blue & $68 \pm 20$ & $9 \pm 7$ & $-18 \pm 10$ \\
Mid & & $14 \pm 1$ & $20 \pm 9$ \\
Red & & $130 \pm 30$ \\
\hline
\end{tabular}

Table 1 reports the auto- and cross-correlations of the top $20 \%$ blue and red galaxies along with the mid $60 \%$. The error bounds in the table are derived from the actual scatter between the two fields, and appear to be twice the statistical, Poisson errors. These results show that the auto-correlations of the intermediate colors are weaker than for the whole data set, and the strong correlations in the two extreme colors boost the overall clustering amplitude to its observed value $18.0 \pm 1.8$. In addition, the cross correlation function 
between the red and blue populations is marginally anti-correlated.

\subsection{Comparison with Models}

We have compared our results to predictions of models (Gronwall \& Koo 1995), designed to fit the observed counts, colors, and redshift distributions, but that rely on having the luminosity function of field galaxies be a free "parameter", i.e., one that is not constrained by local observations. The models include some mild evolution in the stellar populations and a small amount of intrinsic absorption/reddening due to dust. The use of models was not meant to be part of an exhaustive analysis of various evolutionary and cosmological scenarios, but rather to provide vital baseline estimates of the redshift distributions needed to interpret the angular correlations. These models are improved estimates over those of prior analyses of angular correlation functions (Efstathiou et al 1991, Koo \& Szalay 1984), since they are also found to fit a wide range of other faint galaxy counts, colors, and redshifts.

To compare the actual observations, simulated galaxy catalogs were generated that account for photometric errors and incompleteness that mimic those expected to exist in our data. We then applied identical magnitude $\left(20<B_{J}<23.5\right)$ and color cuts $\left(U-R_{F}>1.5\right.$ and $\left.U-R_{F}<-0.25\right)$ to the simulated catalogs and derived the redshift distributions. These were used to calculate the angular correlation amplitude, assuming a standard no-evolution, -1.8 slope correlation function. The models reproduce the overall correlation amplitude accurately (18.2), and can be reconciled with the amplifications seen in the 'red' subsets, but fail to explain the angular clustering of the extremely 'blue' subset,

$$
\begin{array}{r}
A_{w}(\text { blue }) / A_{w}(\text { all })=0.44 \\
A_{w}(\text { red }) / A_{w}(\text { all })=1.67 \\
A_{w}(\text { cross }) / A_{w}(\text { all })=1.02
\end{array}
$$

The discrepancy is so large that no minor modification of the models can give a satisfactory agreement. The redshift distribution for the 'blue' galaxies was more extended than for the whole sample (because of evolution), resulting in a larger number of projected pairs, thus in a lower amplitude for the predicted angular correlation amplitude. Thus the main question is what constraints these observations generate on the redshift distribution of the bluest galaxies.

\subsection{Comparison to Redshift Surveys}

Deep redshift surveys are just reaching our magnitude limits (Colless et al. 1991, 1993; Glazebrook et al. 1995; Lilly et al. 1995). The redshift survey most similar in limiting magnitude to our data is the Glazebrook et al. (1995) survey to $B<24$. This sample has 73 measured redshifts. Taking the 21 bluest galaxies of the $B<23.5$ subset, only 10 have redshifts; thus the incompleteness is over $50 \%$, whereas the whole survey was $\approx 70 \%$ complete. These 10 galaxies were rather evenly distributed over the redshift range of 0.08 to 1.1; thus this blue subset of the spectroscopic sample is too small and incomplete to draw reliable conclusions about the detailed redshift distribution to be drawn when such narrow color divisions are applied. The Lilly et al. (1995) sample to $I<22$ has a larger number of redshifts (600), but these are unpublished. We discuss their result in the Summary. 


\section{Discussion and Analysis}

\subsection{The Red Galaxies}

In some respects, the large enhancement in the correlation amplitude of the reddest galaxies may seem as surprising as that for the blue subset. However, this result can be explained in terms of known properties of red galaxies quite naturally. An enhancement in the correlation amplitude for the red galaxies may be expected since these are primarily early type (E/S0) galaxies (morphology-density relation). Further inspection of the red subsets found in these fields reveals that field SA57 contains a known cluster at $z=0.24$ and SA68 contains a supercluster at a redshift of $z=0.54$, both visible in maps of the fields (Figs. 10 and 11 in Koo 1986). From the models we have estimated that, for galaxies redder than $U-R_{F}>1.5$ (with a median redshift of 0.34 ) the correlation amplitude is enhanced by a factor 1.67 from the modified redshift distribution alone. Since that population is hardly evolving, these model predictions should be quite robust. On the other hand, a 4 times stronger intrinsic clustering has actually been measured for E/S0 galaxies (Nicotra et al. 1993), resulting in a total correlation amplification of 6.4. Therefore, the measured overall enhancement of the red galaxy signal can be well understood in these terms, and illustrates the sensitivity of using angular correlations as a function of color to study both galaxy evolution and large scale structure.

\subsection{Blue Galaxies}

Analyzing the enhancement in the correlation amplitude of the bluest galaxies is more problematic than that of the red subset since these have not been identified securely with a well-studied local population. To reach quantitative conclusions about their redshift distribution, we will exploit two basic observational constraints on blue galaxies which generate opposing trends: (1) the amplification of the very blue galaxy angular correlations is greater than 6 , and (2) the population fraction is greater than $15 \%$. These constraints provide strong limits on the possible redshift distributions.

Several hypotheses have been proposed to explain the nature and distribution of faint blue galaxies. One hypothesis is that faint blue galaxies are the precursors of normal galaxies but undergoing enhanced star formation at an earlier epoch, $z>1$. Another is that these galaxies are a merging population, perhaps at intermediate to high redshifts, since merging galaxies are expected to be fairly bright and blue because of enhanced star formation (Broadhurst, Ellis, \& Glazebrook 1992). Other hypotheses include a dominant population of relatively nearby, low surface brightness galaxies that are not represented in existing estimates of the local luminosity function (McGaugh 1994) and the existence of a local bursting dwarf population with strong luminosity evolution that has presently faded from view (Broadhurst et al. 1988; Babul \& Rees 1992; Eales 1993; Lilly 1993). Below, we discuss these possibilities to assess whether any of these scenarios is consistent with our correlation function measurements.

An enhancement in autocorrelation amplitude for the blue subsample can be explained either by these galaxies being intrinsically more strongly correlated than commonly assumed, or their redshift distribution being very different, or a combination of both. Given that the intrinsic spatial correlation of late-type galaxies has been found to be 4 times 
weaker than that of early type galaxies (Nicotra et al. 1993), arguments that postulate increased correlation amplitudes appear to be untenable. To explain the observed enhancement in $w(\theta)$ solely by this effect would require the opposite trend, namely, a 12 times stronger intrinsic clustering for the very blue subset if they had the redshift distribution as predicted by the model, or 6 times stronger if they had the redshift distribution of the whole sample. This leaves the possibility that the redshift distribution of the bluest galaxies is quite different from the predictions. Since the angular correlation amplitude is proportional to the second moment of $d n / d z$, the most obvious implication of the enhanced clustering amplitude is that the redshift distribution is narrower than the models suggest.

In considering the possibility that the bluest galaxies are all at high redshift, let us assume that our blue galaxies are the distant counterparts of normal galaxies today that have undergone enhanced star formation in the past. Having measured the power-law slope of the correlation function of the blue galaxies to be similar to that of galaxies today, and assuming that these counterparts predominantly lie at high redshifts $z \geq 1$, the most straightforward method to increase the correlation amplitude would be to have a strongly peaked redshift distribution. Such a peaked distribution, though in principle possible is unlikely given the broad shape of the luminosity function, unless special galaxy formation models are invoked, such as by Babul and Ferguson (1995). Furthermore, the redshift that correspondes to the 15 percentile point in the high redshift tail of a standard galaxy distribution is about $z=0.76$ at our magnitude limit. Any subset of this tail will not have enough galaxies to make up the observed $15 \%$.

Another explanation is that the very blue galaxies are predominantly a rapidly merging population at $z \geq 1$. In such a scenario, however, one might expect these galaxies to have a correlation function with an excess of close pairs. While several studies support an increase of close pairs at the level of $(1+z)^{3}$ (Zepf \& Koo 1989; Burkey et al. 1994; Carlberg, Pritchet, \& Infante 1994; Yee \& Ellingson 1995), the fraction of participating galaxies remains small, at the level of $10 \%$ or less. More importantly, very close pairs do not appear to have colors that differ significantly from typical field galaxies. Since we also find that the very blue galaxy correlation function has the same smooth power-law slope as that of the red galaxies, without a break to higher amplitudes on small scales, we consider the merger hypothesis an unattractive explanation. In any case, it is not the existence of close pairs that accounts for the enhancements in our signals.

The other extreme possibility is that the blue galaxies are at low redshift. Indeed, the enhanced correlations suggest this to be the case - in the integral that gives the angular correlation amplitude, the $(d n / d z)^{2}$ term has a very large weight at low redshifts, thus it is much easier to account for the excess clustering by modifying the redshift distribution at the low $z$ end. To quantify this result, without going into an overly complicated modeling scheme, we will try to parameterize the phenomenological redshift distribution of the blue galaxies by a simple functional form. This will show that, independent of the particular details, the only possible solution is to have most of these galaxies at redshifts $z<0.3$.

For this simple parametrization we make the following assumptions: (1) the number counts combined with redshift surveys fix the redshift distribution of the whole sample, with $20<B_{J}<23.5$, (2) the red galaxies in the sample do not evolve, and their redshift 
distribution is adequately described by the models, (3) a standard, non-evolving clustering amplitude is used throughout.

For our first experiment, we try to assign the blue galaxies to as low a redshift as possible. We adopt the following algorithm for selecting the redshift distribution of the blue subset: we subtract the red galaxies from the overall redshift distribution, creating a complementary sample. This subset is multiplied by a simple lowpass window, and the resulting distribution is assigned to the bluest subset. The overall population fraction and amplification of $w(\theta)$ of this subset are calculated. Satisfactory subsets are required to have a fraction greater than $15 \%$, and an amplification greater than 6 . Although this recipe falls short of providing a full modeling of all the color distributions, number counts, and correlations, it is straightforward and incorporates some basic galaxy distribution properties. The window to select the low-redshift galaxies is a simple, sharp low-pass filter, i.e. all galaxies below that redshift are assigned to have such blue colors. This is clearly unrealistic, but this is how we can make the redshifts of the blue galaxies the lowest possible. We find that the highest redshift cutoff with such models is at $z=0.28$.

This simple model is clearly in conflict with the redshift surveys. Glazebrook et al. (1995) found that at least 4 of the 21 bluest galaxies were at redshifts beyond 0.6. Adopting an additional conservative constraint, that at least $25 \%$ of this bluest subset has to be at $z>0.8$, we can do another experiment. We start with the bluest galaxies from the models. Their redshift distribution is quite flat. We add an extra low redshift component from the rest of the galaxies, with a sharp upper cutoff in their redshifts. The highest cutoff satisfying these constraints is at $z=0.2$, in which case $60 \%$ of the galaxies are below $z=0.3$. In this scenario all of the remaining galaxies had to be assigned to the low redshift tail. The other extreme case in such a picture is to assign extra galaxies at very low redshifts, at $z<0.05$. In this case we can get away with adding a small fraction of all the blue galaxies; however, the small fraction necessary for the amplification exceeds the number of available galaxies at that redshift by a factor of 2 .

\section{Conclusion: Low-Redshift Faint Galaxies}

We conclude from these exercises that it is not possible to explain the observed excess correlations with even moderately large modifications of the class of models we have considered, that is, a substantial fraction of the blue galaxies must exist below a redshift of $z=0.3$. However, such analyses alone cannot discriminate between a population of blue galaxies all at redshifts up to 0.3 (e.g. low surface brightness galaxies), versus a strongly evolving and thus more peaked population. However, we can certainly rule out the possibility that the bulk of these galaxies are of high luminosities with a wide spread over high redshifts. From the results of the data and the models, we conservatively predict that more than $50 \%$ of the currently unidentified population of the faint blue galaxies at our magnitude limit will be found at redshifts $z<0.3$.

With evidence that these very blue galaxies are mostly nearby, their luminosities can be estimated by applying a K-correction of 0 . The galaxies in this survey typically have $22<B_{J}<23.5$, which translates to limits on absolute magnitudes of $-18>M_{B_{J}}>-19.5$ at redshifts of 0.3 . For lower redshifts, the derived luminosities will, of course, be fainter. 
Typical $\left(\mathrm{L}^{*}\right)$ galaxies have $M_{J} \sim-21.0$ and thus the very blue galaxies appear to be of low luminosities. If their blue color is due to enhanced star formation activity, so that their luminosity has increased as well, their progenitors may well be from the faint end of the luminosity function.

Since a rapid luminosity evolution of these blue galaxies, combined with our magnitude cutoff at $B_{J}=23.5$, can create a reasonably peaked redshift distribution between 0.2 and 0.3 , a low redshift population would result if the faint end slope of the luminosity function of very blue galaxies were steep, as suggested by the results of Marzke et al. (1994) or Metcalfe et al. (1991). Such a hypothesis is not only supported by our work, but also by recent redshift surveys. Consider the expected color tracks traversed by galaxies of different intrinsic colors as a function of redshift in the Canada-France Redshift Survey (see for example Fig 2 of Lilly et al. (1995) which shows $(V-I)_{A B}$ vs redshift to $\left.I<22\right)$. The exact distribution of these galaxies involves a complex interplay between the depth of the survey, luminosity functions, evolution, cosmology, dust; however, the bluest galaxies are found to be at either very low or very high redshifts. Based on this figure of almost 600 galaxies, we find that the bluest galaxies indeed appear to be concentrated at low redshifts. More specifically, reading off the uncrowded bluest 18 points, 11 are below $z=0.3$, just as we have predicted with our correlation analysis. The strongly evolving luminosity function of the bluest half in this data set also suggests that these effects are even more pronounced for the bluest 15\%. Therefore, we conclude that the faint end slope of the local luminosity function for blue galaxies is steeper than that adopted by our baseline model and that perhaps the luminous component at high redshifts is less. Thus this redshift survey supports our work and shows the effectiveness of our approach. Of the 10 bluest galaxies with redshifts in the Glazebrook et al. (1995) sample, 4 had redshifts below $z=0.3$, and 4 were above 0.6 . The remaining 11 are a large enough percentage that their result may still be consistent with ours. We predict that these remaining objects will be found to be low-metallicity systems with redshifts less than 0.3 .

Having identified the blue galaxies as being dominated by a low redshift population, we can estimate the expected strength of the cross-correlation between the red and blue subsets. From the models - with the assumption that the intrinsic clustering is average we obtain expected amplifications between 0.7 and 1.3 , which yields an amplitude range of $18 \pm 15$. Contrary to this expectation is the observed value $-18 \pm 10$ shown in Table 1 , a discrepancy of $2 \sigma$. However, our null hypothesis of average clustering is likely to be incorrect; unfortunately no local information is available on the intrinsic, spatial crosscorrelations of different galaxy types. This measured amplitude is marginally consistent with a null signal. If the numbers are taken literally, we may speculate that the weak anticorrelations indicate that only a small fraction of the very blue galaxies can co-exist with the red galaxies at similar, moderate redshifts, and for those that do co-exist, they occupy separate environments. Since red, early type galaxies populate high density regions, the faint blue galaxies should be in surrounding, segregated lower-density regions, which is not inconsistent with common beliefs. The primary limitation in this analysis, which prevented us from being able to make much stronger statements about the redshift distribution of these faint galaxies, is the poor knowledge of the local auto-and cross correlation properties of the different galaxy types. 
We have shown that angular correlations as a function of color can serve as a very sensitive statistical tool to study galaxy evolution as well as to provide new insight into the nature of galaxies of extreme colors. Since multicolor photometric observations easily reach beyond the practical limits of reliable redshift measurements, angular correlation functions, combined with color cuts, will play an increasingly important role in understanding faint galaxies in the future. As the local galaxy population is better understood, the accuracy of this method will increase substantially.

\section{Acknowledgements}

The authors thank the several referees including Karl Glazebrook for useful suggestions, C. Gronwall for providing the model catalogs for our simulations, S. Majewski for providing various stellar catalogs for checking against systematic effects, and N. Roche for useful discussions. David Koo and Alex Szalay have been supported by an NSF/Hungary exchange grant, and by a grant from the US-Hungary Joint Fund. Alex Szalay has been supported by NSF grant AST 90-20380 and David Koo by AST 88-58203. Stephen Landy has been supported both by the Johns Hopkins University and an NSF grant at Carnegie Observatories.

\section{References}

Babul,A. \& Rees,M.J. 1992, MNRAS, 255, 346

Babul,A. \& Ferguson,H. 1995, ApJ, , submitted

Bernstein, G. M., Tyson, J. A., Brown, W. R., \& Jarvis, J.F. 1994, ApJ, 426, 516

Brainerd,G.T., Smail,I., \& Mould,J.R. 1995, MNRAS, submitted, .

Broadhurst,T.J., Ellis, R.S. \& Shanks,T. 1988, MNRAS, 235, 827

Broadhurst,T.J., Ellis, R.S. \& Glazebrook,K. 1992, Nature, 355, 55

Burkey,J.M., Keel,W.C., Windhorst,R.A., \& Franklin,B.E. 1994, ApJ, 150, L13

Carlberg,R.G., Pritchet,C. J.,\& Infante, L. 1994, ApJ, 435, 540

Colless,M.M. 1994, in Wide Field Spectroscopy and the Distant Universe, ed. S. Maddox, World Scientific, 263.

Colless,M.M., Ellis,R.S., Broadhurst,T.J.,Taylor, K. \& Peterson,B.H. 1993, MNRAS, 261, 19

Colless,M.M., Ellis,R.S., Taylor,K., \& Shaw,G. 1991, MNRAS, 253, 686

Cowie,L.L.,Songaila,A. \& Hu,M. 1991, Nature, 354, 460

Eales,S. 1993, ApJ, 404, 51

Efstathiou, G.P.E. 1995, MNRAS, 272, L25

Efstathiou,G.P.E., Bernstein,G., Katz,N., Tyson,J.A. \& Guhathakurta,P. 1991, ApJ, 380, L47

Giovanelli,R., Haynes,M.P. \& Chincarini,G.L. 1986, ApJ, 300, 77

Glazebrook,K., Ellis,R.S., Colless,M.M., Broadhurst, T.J., Allington-Smith,J.R., Tanvir,N.R. \& Taylor,K. 1995, MNRAS, 273, 157 
Griffiths,R.E., Casertano,K.U., Ratnatunga,L.W., Neuschaefer,L.W., Ellis,R.S., Gilmore,G.F., Glazebrook,K., Santiago,B., Huchra, J.P., Windhorst,R.A., Pascarelle,S.M., Green,R.F., Illingworth,G.D., Koo,D.C. \& Tyson,A.J. 1994, ApJ, 435, L19

Gronwall,C. \& Koo,D.C. 1995, ApJ, 440, L1.

Infante,L. \& Pritchet,C.J. 1992, ApJS, 83, 237

Infante,L. \& Pritchet,C.J. 1995, ApJ, 439, 565

Jones,L.R., Shanks,T., Fong,R. 1988, in Large Scale Structures of the Universe, IAU Symp. 130 ed. J. Audouze, M.C. Pelletan, A.S. Szalay (Dordrecht:Kluwer), 528.

Koo, D. C. 1996, in Examining the Big Bang and Diffuse Background Radiations,IAU Symp. No. 168, ed. M. Kafatos (Kluwer: Dordrecht), in press

Koo, D.C. 1986, ApJ, 311, 651

Koo, D.C., \& Kron, R. G. A. 1992, Rev. ARA\&A, 30, 613

Koo, D.C., \& Szalay, A. S. 1984, ApJ, 282, 390

Kron, R.G. 1980, ApJS, 43, 305

Landy, S.D., \& Szalay, A.S. 1993, ApJ, 412, 64

Lilly, S.J. 1993, ApJ, 411, 501

Lilly, S.J., LeFerre, O., Hammer, F., Crampton, D., \& Tresse,L. 1995, in Wide Field Spectroscopy of the Distant Universe, (Singapore:World Scientific)

Marzke,R.O., Geller,M.J., Huchra,J.P. \& Corwin,H.G. 1994, AJ, 108, 437

Metcalfe,N., Shanks,T., Fong,R.\& Jones,L.R. 1991, MNRAS, 249, 498

McGaugh,S. 1994, Nature, 367, 538

Neuschaefer,L.W., \& Windohirst,R.A. 1995, ApJ, 439, 14

Nicotra,M.A., Abadi,M.G.,\& Lambas,D.G. 1993, in Observational Cosmology ed. G.Chincarini, A.Iovino, T.Maccacaro and D. Maccagni (ASP Conference Series, Vol 51 1993), 152.

Neuschaefer,L.W., Ratnatunga,K.U., Griffiths,R.E., Casertano,S. \& Im,M. 1995, ApJ, , in preparation

Peebles,P.J.E. 1980 in The Large Scale Structure of the Universe, (Princeton Univ. Press, Princeton)

Pritchet,C.J. \& Infante,L. 1992, ApJ, 399, L35

Stevenson,P.R.F., Shanks,T., Fong,R. \& MacGillivray,H.T. 1985, MNRAS, 213, 953

Yee,H.S. \& Ellingson,E. 1995, ApJ, , in press

Zepf,S.E. \& Koo,D.C. 1989, ApJ, 337, 34 


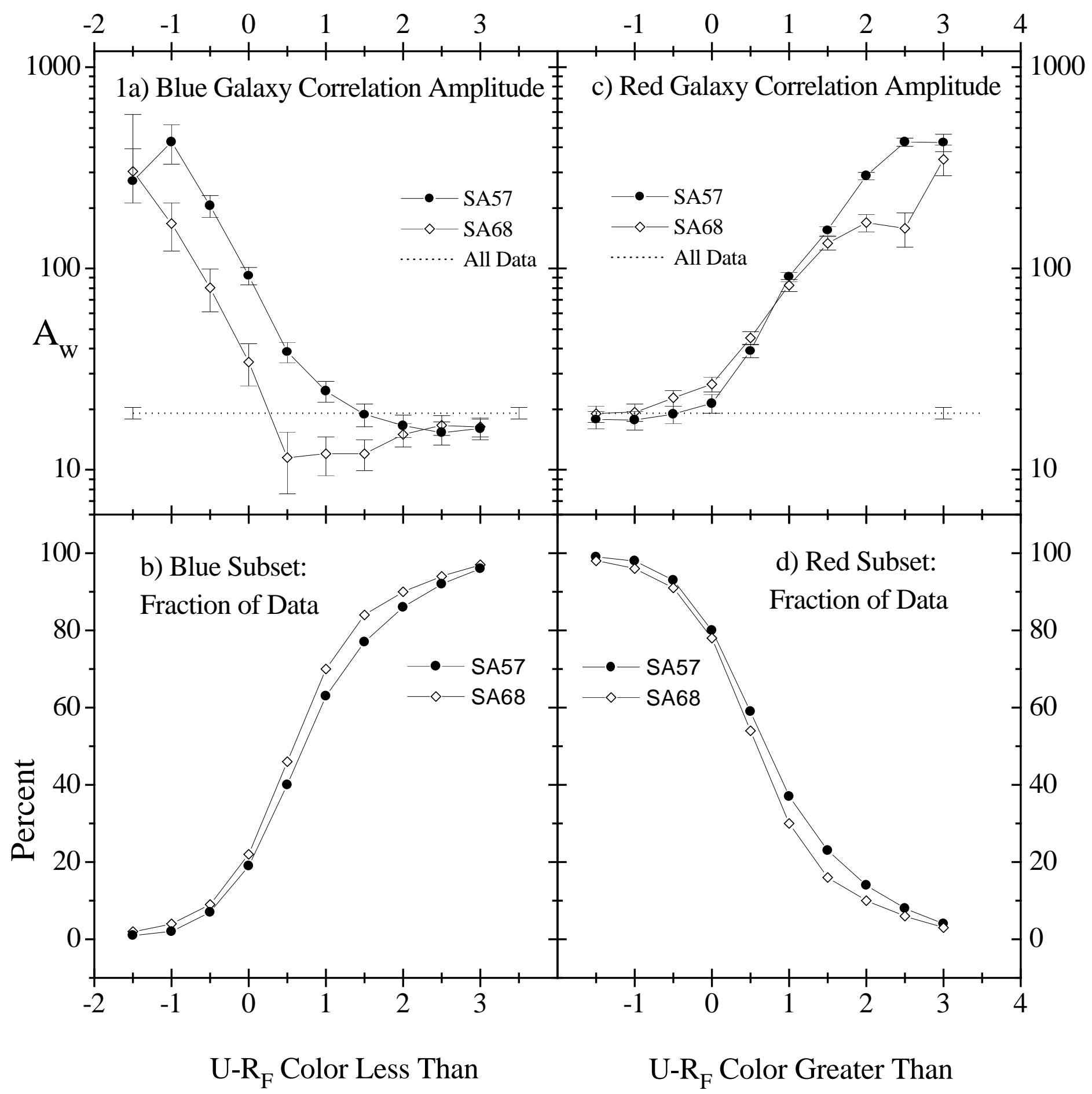

Fig. 1) Results for $A_{w}$ as a function of limiting color assuming a power law slope of -0.8 are shown, integrated between 9 and 144 arcseconds for both fields. The signals are calculated using all galaxies less than or greater than a given $\mathrm{U}-\mathrm{R}_{\mathrm{F}}$ color for the blue and red respectively. Error bars are Poisson in the bin counts (although see Section 4.1). The dashed line shows the result for the combined data sets with no cut in color. Notice the enhancements over a factor of 10 for both the extreme blue and red subsets. The fraction of the entire data set used at each limit is shown below each plot in b\&d. 


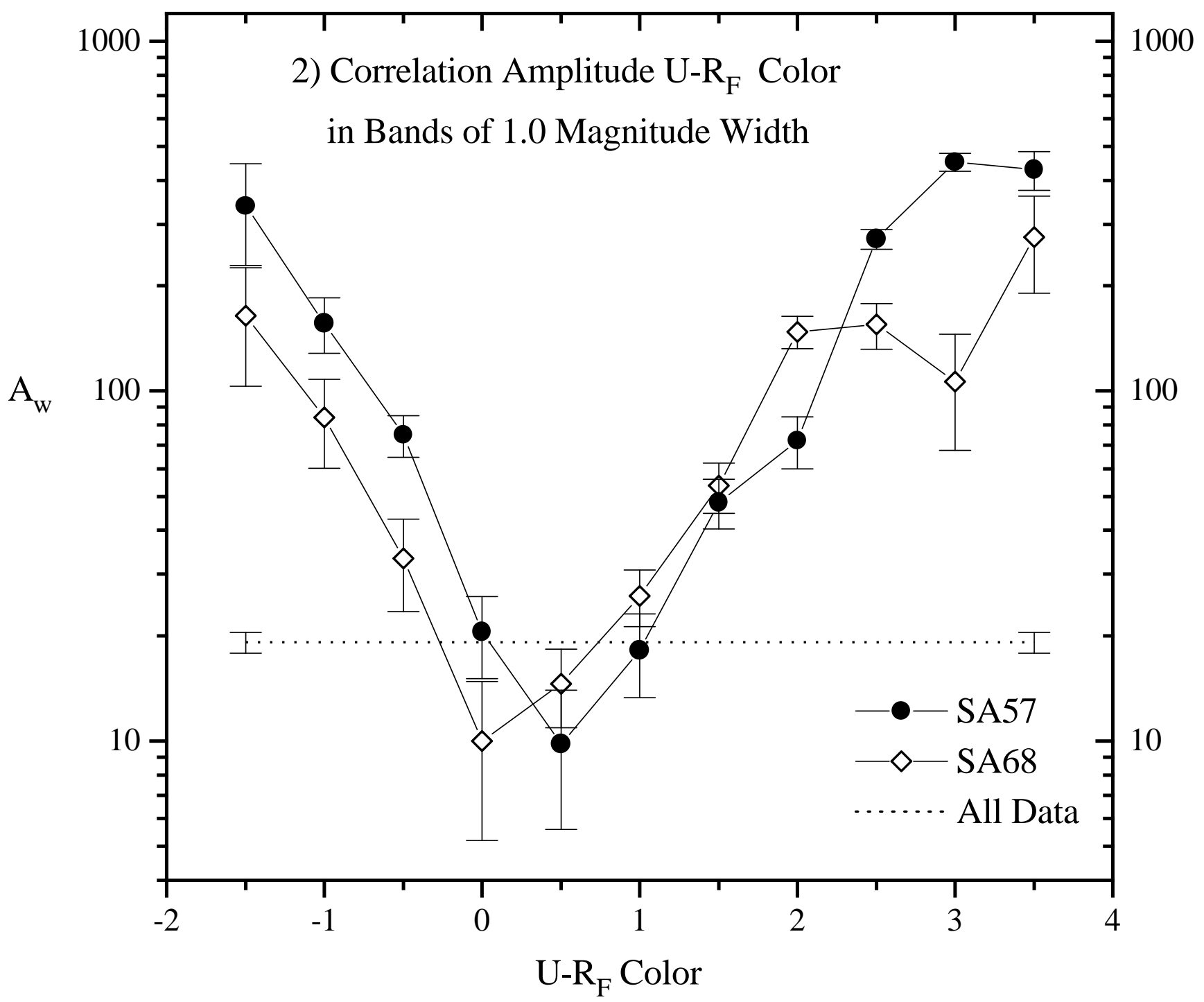

Fig 2) Results for $A_{w}$ with the data sets divided into bands of $\mathrm{U}-\mathrm{R}_{\mathrm{F}}$ color of one magnitude width, assuming a power law slope of -0.8 , and integrated between 9 and 144 arcseconds for both fields. These results show that the enhancements in both blue and red are robust over 1.5 magnitudes in $\mathrm{U}-\mathrm{R}_{\mathrm{F}}$. The dashed line shows the result for the combined data sets with no cuts in color. 


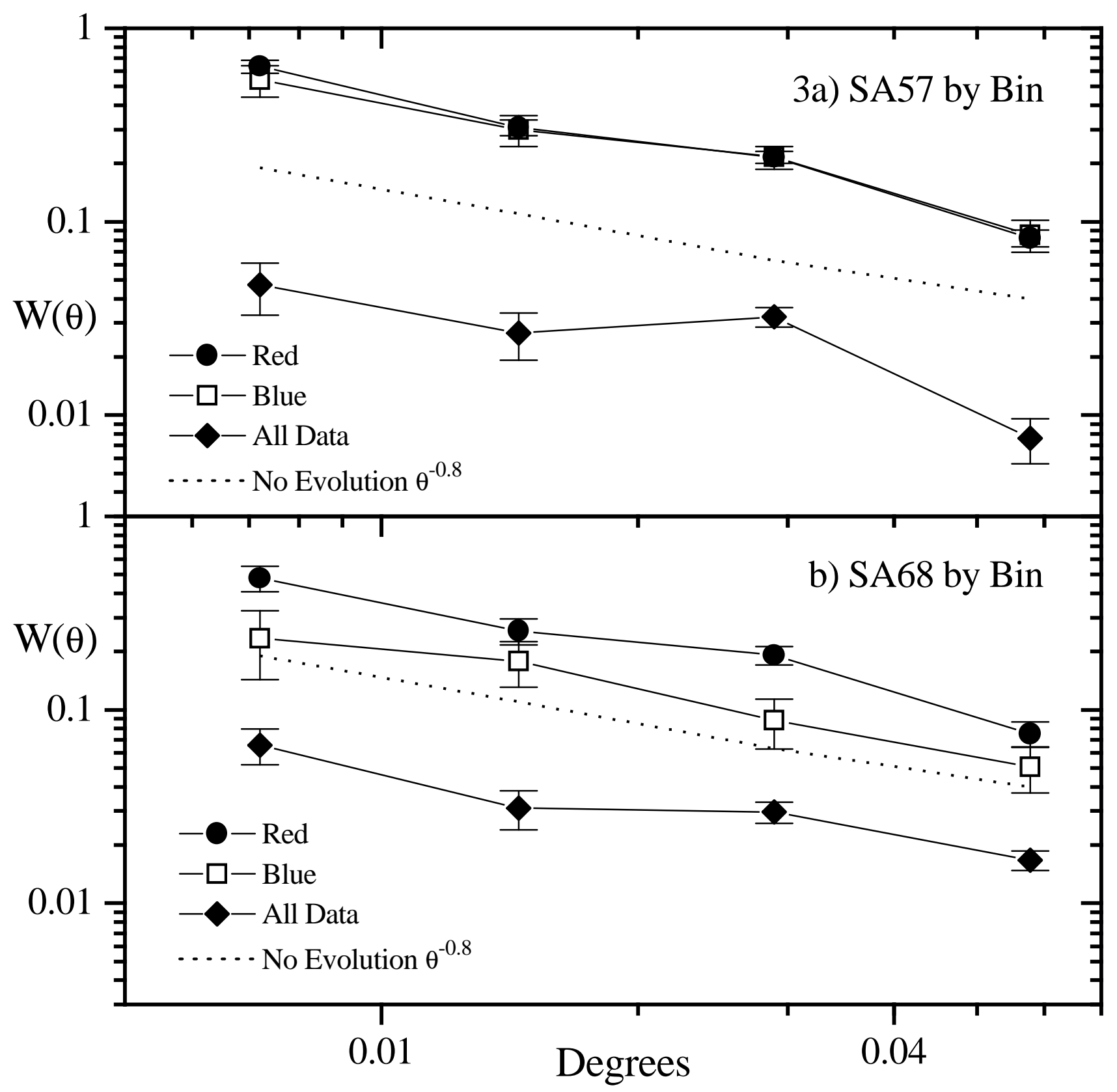

Fig 3) The angular correlation function $\mathrm{W}(\theta)$ for $\mathrm{U}-\mathrm{R}_{\mathrm{F}}>1.5$ (Red) and $\mathrm{U}-\mathrm{R}_{\mathrm{F}}<-0.25$ (Blue) for both data sets broken up into 5 bins of equal log width between 18 and 289 arcseconds along with a fudicial line for a no-evolution model with $\mathrm{q}_{\mathrm{o}}=0.01$ and $\mathrm{H}_{\mathrm{o}}=50 \mathrm{~km} \mathrm{sec}^{-1} \mathrm{Mpc}^{-1}$. The results for both fields in both colors are consistent with the canonical power law slope of -0.8 . 